\title{
On fractionality of the path packing problem
}

\author{
Natalia Vanetik*
}

\begin{abstract}
In an undirected graph $G$ with node set $N$ and a subset $T \subseteq N$, a fractional multiflow problem is defined as finding $\max _{f} \sum_{(u, v)} \omega(u, v) f[u, v]$ over all collections $f$ of weighted paths with ends in $T$ (the $\omega$-problem). $f[u, v]$ denotes the total weight of paths with the end-pair $(u, v)$ in $f$. The paths of $f$ must satisfy the edge capacity constraint: total weight of the paths traversing a single edge does not exceed 1 . We study a fractional multiflow problem with the reward function $\omega$ having values $(0,1)$ (a fractional path packing problem), and an auxiliary weak problem where $\omega$ is a metric. A. Karzanov in [ $\mathrm{K}$ 1989] defined the fractionality of $\omega$ with respect to a given class of networks $(G, T)$ as the least natural $D$ such that for any network $(G, T)$ from the class, the $\omega$-problem has a solution which becomes integer-valued when multiplied by $D$. He proved that a fractional path packing problem has infinite fractionality outside a very specific class of networks, and conjectured that within this class, the fractionality does not exceed 4 (2 for Eulerian networks). In this paper we prove Karzanov's conjecture by showing that the fractionality of both fractional path packing and weak problems is 1 or 2 for every Eulerian network in this class.
\end{abstract}

\section{Introduction}

In this paper we study collections of edge-disjoint paths in a network, also called paths packings or multiflows, addressing an optimization problem of the following form. Let $G=(N, E)$ be a multigraph with node-set $N$ and edge-set $E$, and let $T \subseteq N$ be a set of nodes distinguished as terminals. By a T-path we mean an unclosed path with the ends in T, and by an integer T-flow, or an integer multiflow, we mean a collection of pairwise edge-disjoint $T$-paths in $G$. Let us define a fractional $T$-flow as a non-negative weight function $f(P)$ on the set of all $T$-paths in $(G, T)$, satisfying the edge capacity constraints:

$$
\sum_{P} f(P) I(P,(x, y)) \leq c(x, y) \text { for each adjacent pair }(x, y) \text { of nodes in } N
$$

\footnotetext{
*Department of Computer Science, Ben-Gurion University, Israel, orlovn@cs.bgu.ac.il
} 
Here $I(P,(x, y))$ denotes the number of $(x, y)$-edges of $G$ traversed by $P$, and $c(x, y)$ is the edge capacity, equal to the number of $(x, y)$-edges in $G$. Given non-negative "rewards" $\omega(u, v)$ assigned to the unordered pairs of terminals, the problem is to

$$
\text { maximize } \sum_{u, v} \omega(u, v) f[u, v] \text { over the fractional } T \text {-flows } f \text { in }(G, T) \text {, }
$$

where $f[u, v]$ denotes the total weight of the $(u, v)$-paths in $f$. For short, (1.2) will be referred to as the $\omega$-problem. This is one of the basic multiflow problems, having numerous applications, such as communication and VLSI design. Not surprisingly, for most reward functions the $w$ problem is known to be $\mathbb{N P}$-hard over integer multiflows, not only when a network $(G, T)$ is quite arbitrary, but even for such friendly classes as the planar or the Eulerian networks (the latter class is studied in this paper).

However, the more fragmented is $f$ between various paths, the less is its utility for discrete path packing. To make this precise, let us, following A. Karzanov [ $\mathrm{K}$ 1989], define the fractionality of the reward function $\omega$ with respect to a given class of networks $(G, T)$ : this is the least natural $D$ such that for any network $(G, T)$ from the class, the $\omega$-problem has a solution $f$ which becomes integer-valued when multiplied by $D$ (in short, a $\frac{1}{D}$-integer solution). For certain reward functions, fractionality for the general networks was found to be 2 (see [IKL 2000] and [L 2004]); for some of them, the $\omega$-problem was also shown to have an integer solution provided that the non-terminal (inner) nodes of a network have even degrees; such networks are called Eulerian.

Two specific classes of the reward function are of principal importance. One comprises the $(0,1)$ reward functions. It is convenient to represent such a function by a demand graph (or scheme) $(T, S)$ where $S:=(u, v): \omega(u, v)=1$, and to call (1.2) the S-problem. Let a path in $G$ be called an $S$-path if its end-pair belongs to $S$, and a collection of $S$-paths satisfying (1.1) be called an $S$-flow. Thus, the $S$-problem may be stated as maximizing of

$f[S]:=\sum_{(u, v) \in S} f[u, v]$. A. Karzanov has described the fractionality of the $(0,1)$ reward functions (or the schemes $S$ ) in [K 1989]. Namely, the fractionality of $S$ is finite iff any distinct pairwise intersecting anticliques (i.e., inclusion-maximal stable sets) $A, B, C$ of $(T, S)$ satisfy

$$
A \cap B=A \cap C=B \cap C
$$

and the finite fractionality can only equal 1, 2, or 4 . He conjectured that this 


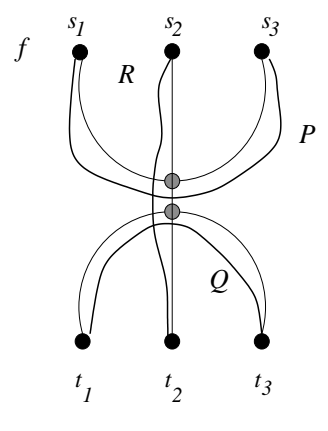

Integer solution
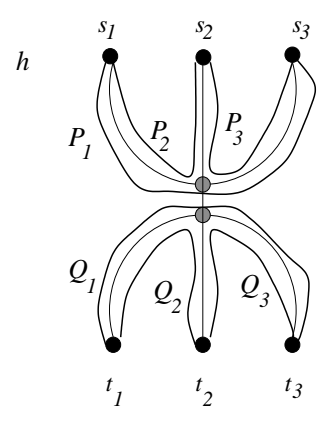

Half-integer solution

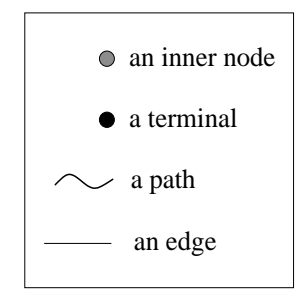

Figure 1: The fractionality of $S$-problem and $W$-problem can be 2.

Not long ago, H. Ilani and E. Barsky observed that the problem of discrete path packing is $\mathbb{N P}$-hard, even for Eulerian networks, for each demand graph violating (1.3). So, investigating the $S$-problem has focused on the schemes satisfying (1.3). In this paper we consider the $S$ problem for $S$ satisfying (1.3) together with an auxiliary weak problem, denoted a $W$-problem: an $\omega$-problem where $\omega$ is a metric defined by $\omega(u, v)=1$ for $(u, v) \in S, \frac{1}{2}$ for $(u, v)$ covered by exactly one anticlique of $(T, S)$, and 0 for the others (i. e., those covered by at least two anticliques). An anticlique clutter of $(T, S)$ satisfying (1.3) is called a $K$-clutter, and an Eulerian network $(G, T, \mathcal{K})$ with an anticlique $\mathrm{K}$-clutter $\mathcal{K}$ of $(T, S)$ is called a $K$-network. The maxima of $S$ - and $W$-problems are denoted by $\eta$ and $\theta$ respectively.

In this paper, we prove conjecture (1.4). Additionally, we show that the $W$-problem in a $\mathrm{K}$ network also admits a solution of fractionality at most 2 . We use the following crucial fact: every $S$-problem and $W$-problem in a network satisfying (1.3) have a common solution (Theorem 1 of [Va 2007]).

The bound on fractionality is tight in both cases, as an example in Figure 1 demonstrates. There we have $\mathcal{K}=\left\{\left\{s_{i}, t_{j}\right\}\right\}, i, j \in\{1,2,3\}$, and every integer multiflow in this network has no more than $2 S$-paths, for example, paths $P$ and $Q$ in Figure 1(a). The maximum of the $W$-problem among integer multiflows is $2 \frac{1}{2}$. However, in this network there exists a half-integer multiflow $h=\left\{P_{1}, P_{2}, P_{3}, Q_{1}, Q_{2}, Q_{3}\right\}$ with weight of every path $\frac{1}{2}$ being (see Figure $1(\mathrm{~b})$ ). The value of $\sum_{u, v} \omega(u, v) h[u, v]$ for both $S$-problem and $W$-problem is 3 . Thus, an integer solution to the $S$-problem or the $W$-problem does not always exist. Table 1 summarizes notation used in this paper. 


\begin{tabular}{|l|l|}
\hline Notation & Definition \\
\hline \hline$(G, T, \mathcal{K})$ & a network $(G,(T, S))$ and the anticlique clutter $\mathcal{K}$ of $(T, S)$ \\
\hline$S$-path & a path whose end-pair is in $S$ \\
\hline$W$-path & a path whose end-pair is covered by exactly one member of $\mathcal{K}$ \\
\hline zero path & a path whose end-pair is covered by two members of $\mathcal{K}$ \\
\hline$d(X), X \subset N$ & the number of $(X, \bar{X})$-edges in $G$ \\
\hline$\lambda(A), A \subseteq T$ & min $\{d(X): X \subset N, \quad X \cap T=A\}$ \\
\hline$\beta(A), A \subseteq T$ & $\frac{1}{2}\left(\sum_{t \in A} \lambda(t)-\lambda(A)\right) ;$ is an integer in Eulerian networks \\
\hline$A^{c}, A \subseteq T$ & $T \backslash A$ \\
\hline $\bar{A}, A \subseteq N$ & $N \backslash A$ \\
\hline an $(A, B)$-path (an $A$-path), $A, B \subseteq N$ & a path ends in $A$ and $B$ (in $A)$ \\
\hline$f[A, B]$ & the number of $(A, B)$-paths in $f(f[A]$ when $A=B)$ \\
\hline$w(P)$ & the weight of path $P$ \\
\hline$x P y$ & an $(x, y)$-segment of a path $P$, where $x$ and $y$ are nodes \\
\hline$|f|$ & the size of a multiflow $f:$ the total weight of its paths \\
\hline a maximum multiflow & a multiflow of maximum size \\
\hline the fractionality of a multiflow & the largest denominator among its paths' weights \\
\hline$s \sim t, s, t \in T$ & $(s, t)$ is a zero pair \\
\hline an atom & a set of terminals not separated by a member of $\mathcal{K}$ \\
\hline $\mathcal{K}$ is simple & every atom in $\mathcal{K}$ has size 1 \\
\hline
\end{tabular}

Table 1: Notation

\section{Outline of the proof}

We observe K-networks that are counterexamples to the fractionality conjecture for either $W$ or $S$-problem. First, we prove the fractionality conjecture for the $W$-problem by showing that a half-integer simple multiflow of the smallest size solving the $W$-problem exists. Second, we observe a minimal K-network that fails to satisfy the $S$-problem fractionality conjecture and show that it admits a half-integer solution.

\section{Operations on paths and locking}

A pair of paths with disjoint end-pairs and a common node forms a cross. A path is compound if it traverses a terminal different from its ends, and simple otherwise. A multiflow is called 
simple if it contains only simple paths.

Let paths $P$ and $Q$ of a multiflow $f$ traverse an inner node $x$, so that $P=P^{\prime} x P^{\prime \prime}$ and $Q=Q^{\prime} x Q^{\prime \prime}$. Switching $P$ and $Q$ in $x$ transforms them into $K=P^{\prime} x Q^{\prime}$ and $L=P^{\prime \prime} x Q^{\prime \prime}$ and $f$ into the multiflow $f \backslash\{P, Q\} \cup\{K, L\}$. A split of an inner node $x$ is a graph transformation consisting of removal of $x$ and linking its neighbors by $\frac{d(x)}{2}$ edges so as to preserve their degrees. Given a multiflow $h$ in a network, an $h$-split of an inner node is a split preserving the paths of $h$.

A maximum multiflow $f$ locks a set $A \subseteq T$ if it contains a maximum $\left(A, A^{c}\right)$-flow, that is, if $f\left[A, A^{c}\right]=\lambda(A)$. Otherwise, $f$ unlocks $A$. In other words, $f$ locks $A$ if it contains the smallest possible number of $A$-paths. A. Karzanov and M. Lomonosov have introduced in [KL 1978] the following application of the Ford-Fulkerson augmenting path procedure, assuming that a multiflow traverses each edge. A maximum multiflow unlocks $A \in \mathcal{K}$ if and only if it contains an augmenting sequence $P_{1}, x_{1}, \ldots, x_{i-1} P_{i} x_{i}, \ldots, P_{n}$ of paths $P_{1}$ (an $A$-path), $P_{2}, \ldots, P_{n-1}\left(\left(A, A^{c}\right)\right.$ paths) $P_{n}$ (an $A^{c}$-path) and inner nodes $x_{1}, \ldots, x_{n-1}$ so that $x_{i} \in P_{i}, P_{i+1}$ for $i \in 1, \ldots, n-1$ and $x_{i}$ is located on $P_{i}$ between $x_{i-1}$ and the $A$-end of $P_{i}$. In the paper, we use the fact that unlocking a member of $\mathcal{K}$ and existence of the alternating sequence are equivalent. When $\mathcal{K}$ is a K-clutter, there exists a series of switches of $P_{1}, \ldots, P_{n}$ in $x_{1}, \ldots, x_{n-1}$ that creates a maximum multiflow $f^{\prime}$ containing a cross and having $\Theta\left(f^{\prime}\right) \geq \Theta(f)$. If $f$ solves the $W$-problem and unlocks $A \in \mathcal{K}$, switching $P_{1}, \ldots, P_{n-1}$ in $x_{1}, \ldots, x_{n-2}$ creates a multiflow $f^{\prime}$ with $A$-path $P_{0}^{\prime}$ and $A^{c}$-path $P_{1}^{\prime}$ having a common node $x_{n-1}$, so that every switch of $P_{0}^{\prime}$ and $P_{1}^{\prime}$ in $x_{n-1}$ preserves $\Theta\left(f^{\prime}\right)=\theta$.

Let $P$ and $Q$ be an $A$ - and $A^{c}$-paths of a multiflow $h$ with a common inner node so that $w(P)=$ $w(Q)$ and no switch of $P$ and $Q$ changes $\Theta(h)$. Let us denote the ends of $P$ and $Q$ by $p_{1}, p_{2}$ and $q_{1}, q_{2}$ respectively. Let w.l.o.g. $\left(p_{1}, p_{2}\right),\left(p_{1}, q_{1}\right),\left(p_{1}, q_{2}\right) \in W,\left(p_{2}, q_{1}\right),\left(p_{2}, q_{2}\right),\left(q_{1}, q_{2}\right), \in S$. A multiflow transformation that replaces $P$ and $Q$ with three $\left(p_{2}, q_{2}\right)$-, $\left(p_{2}, q_{2}\right)$ - and $\left(q_{1}, q_{2}\right)$-paths of weight $\frac{w(P)}{2}$ (see Figure 2), is called a $\frac{3}{2}$-operation. It preserves $\Theta(h)$ and increases $h[S]$ by $\frac{w(P)}{2}$.

\section{Fractionality of the $W$-problem}

To prove the fractionality conjecture for the $W$-problem, we show the following:

Theorem 4.1 In every $K$-network $(G, T, \mathcal{K})$ there exists a simple $W$-problem solution of the 

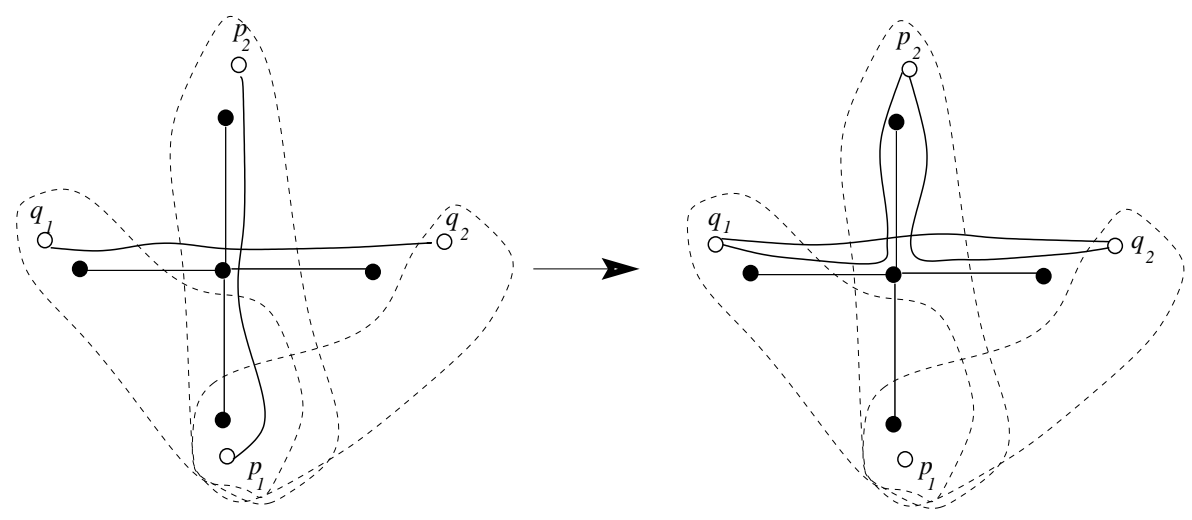

Figure 2: The $\frac{3}{2}$-operation.

smallest size that is half-integer.

We later use this Theorem to prove the fractionality conjecture for the $S$-problem. Let us observe a K-network $(G, T, \mathcal{K})$ which is a minimal counterexample to Theorem 4.1. We assume that $(G, T, \mathcal{K})$ has inner node degree 4 , by the known reduction (see, e.g. [F 1990]), is simple (since atom compression preserves all $W$-problem solutions) and is minimal first in fractionality $k$ of the smallest size $W$-problem solution, and then in $E$ as a set. Then $k=4$, for otherwise we can duplicate each edge in $E$ and obtain a network with $W$-problem fractionality $\left\lceil\frac{k}{2}\right\rceil$. In this section, $f$ denotes a quarter-integer simple multiflow of the smallest size solving the $W$-problem in $(G, T, \mathcal{K})$. For simplicity, we assume that the paths of $f$ have weight $\frac{1}{4}$. Let us denote

$$
\hat{\eta}:=\text { maximum of the } S \text {-problem among simple multiflows in }(G, T, \mathcal{K}) \text {. }
$$

In the Appendix we prove the max-min theorem for the $W$-problem in Theorem [7.1, which implies that for every K-network $(G, T, \mathcal{K}), 2 \theta(G, T, \mathcal{K}) \in \mathbb{N}$ and $2 \hat{\eta} \in \mathbb{N}$. We use these facts in the proof.

\subsection{General flow properties}

Here, we study the behavior of $W$-problem solutions inside the members of $\mathcal{K}$. The series of properties below directly follows directly from the results of Lovãsz, Cherkassky and Lomonosov described in Section 3 ,

Claim 4.2 Let $(G, T, \mathcal{K})$ be a simple $K$-network, and let $h$ be a simple multiflow of fractionality $k$ in it such that $h[A]<\beta(A)$ for some $A \in \mathcal{K}$. Then there exists a simple multiflow $h^{\prime}$ of fractionality $k$ having $\Theta\left(h^{\prime}\right) \geq \Theta(h)+\frac{1}{2}(\beta(A)-h[A])$. 
Proof. Since $h\left[A, A^{c}\right] \leq \lambda(A)$ by definition, and

$$
h[A]=\frac{1}{2}\left(\sum_{t \in A} h\left[t, t^{c}\right]-h\left[A, A^{c}\right]\right)<\frac{1}{2}\left(\sum_{t \in A} \lambda(t)-\lambda(A)\right)=\beta(A),
$$

$\sum_{t \in A} h\left[t, t^{c}\right]<\sum_{t \in A} \lambda(t)$. We modify $h$ by adding paths starting in $t \in A$ until $h\left[t, t^{c}\right]=$ $\lambda(t)$ for all $t \in A$. Since we use edges not saturated by $h$, we obtain a simple multiflow of fractionality $k$, denoted $h^{\prime}$. If $W$ - or $S$-paths of total weight no less than $\beta(A)-h[A]$ were added, $h^{\prime}$ is the required multiflow. Otherwise, some of these paths are cycles that traverse one terminal from $A$ each. Let us modify $h^{\prime}$ into a multiflow without cyclic paths traversing terminals from $A$ using Cherkassky procedure, and denote the resulting multiflow by $h^{\prime \prime}$. If $\Theta\left(h^{\prime \prime}\right) \geq \Theta(h)+\frac{1}{2}(\beta(A)-h[A])$, we are done. Otherwise, we have $\sum_{t \in A} h^{\prime \prime}\left[t, t^{c}\right]=\sum_{t \in A} \lambda(t)$ and $h^{\prime \prime}[A]<\beta(A)$, thus $h^{\prime \prime}\left[A, A^{c}\right]>\lambda(A)$ - a contradiction.

Corollary 4.3 Let $(G, T, \mathcal{K})$ be a simple K-network, and let $h$ be a simple multiflow of the smallest size solving the $W$-problem in $(G, T, \mathcal{K})$. Then $h$ locks $\mathcal{K}$.

Proof. By Claim 4.2, $h[A] \geq \beta(A)$ for all $A \in \mathcal{K}$. If $h$ unlocks some $A \in \mathcal{K}$, i.e. has $h[A]>\beta(A), h$ contains an augmenting sequence for $A$. Switching paths of this sequence creates a simple multiflow $h^{\prime}$ that has the same size as $h$, solves the $W$-problem and allows us to perform a $\frac{3}{2}$-operation, which preserves $\Theta\left(h^{\prime}\right)$ but decreases the size of $h^{\prime}$ - a contradiction.

\subsection{Proof of the weak fractionality theorem}

Let us denote by $\left(G^{\prime}, T^{\prime}, \mathcal{K}^{\prime}\right)$ a network obtained from $(G, T, \mathcal{K})$ by split-offs in one or more inner nodes. We denote the $W$-problem maximum in $\left(G^{\prime}, T^{\prime}, \mathcal{K}^{\prime}\right)$ by $\theta^{\prime}$, and let $A^{\prime}$ and $t^{\prime}$ denote a clutter member and a terminal corresponding to some $A \in \mathcal{K}$ and $t \in T$. We let $g$ denote a simple half-integer $W$-problem solution of the smallest size in $\left(G^{\prime}, T^{\prime}, \mathcal{K}^{\prime}\right) . g$ exists because $(G, T, \mathcal{K})$ is minimal in $E$. Let us denote the value of (4.5) in $\left(G^{\prime}, T^{\prime}, \mathcal{K}^{\prime}\right)$ by $\hat{\eta}^{\prime}$. Note that

$$
\hat{\eta}^{\prime} \leq \hat{\eta}
$$

because by Theorem 1 from Va 2007 $f$ solves the $S$-problem in a network obtained from $(G, T, \mathcal{K})$ by splitting every terminal $t$ into $d(t)$ equivalent terminals of degree 1.

For this type of networks we prove the following series of claims.

Claim 4.4 Let $\theta^{\prime}=\theta-\frac{1}{2}$ and $\hat{\eta}-\hat{\eta}^{\prime} \leq 1$. Then $\sum_{A^{\prime} \in \mathcal{K}^{\prime}} \beta\left(A^{\prime}\right) \leq \sum_{A \in \mathcal{K}} \beta(A)$. 
Proof. Let us assume that $\sum_{A^{\prime} \in \mathcal{K}^{\prime}} \beta\left(A^{\prime}\right)>\sum_{A \in \mathcal{K}} \beta(A)$. As all $\beta(A)$ and $\beta\left(A^{\prime}\right)$ are integers by definition, we have

$$
\theta-\theta^{\prime}=\frac{1}{2}=\hat{\eta}-\hat{\eta}^{\prime}+\left(\sum_{A \in \mathcal{K}} \beta(A)-\sum_{A^{\prime} \in \mathcal{K}^{\prime}} \beta\left(A^{\prime}\right)\right)
$$

thus

$$
1 \geq \hat{\eta}-\hat{\eta}^{\prime}=\frac{1}{2}+\sum_{A^{\prime} \in \mathcal{K}^{\prime}} \beta\left(A^{\prime}\right)-\sum_{A \in \mathcal{K}} \beta(A)>1
$$

a contradiction.

Corollary 4.5 Let $\theta^{\prime}=\theta-\frac{1}{2}$ and $\hat{\eta}-\hat{\eta}^{\prime} \leq 1$. Then for all $A \in \mathcal{K}, \beta\left(A^{\prime}\right) \geq \beta(A)$.

Proof. Let $\beta\left(A^{\prime}\right)<\beta(A)$. Then by Claim 4.2, $g$ can be completed to a half-integer simple flow $g^{\prime}$ in $(G, T, \mathcal{K})$ with $\Theta\left(g^{\prime}\right)=\theta$. Since $|g|=\hat{\eta}^{\prime}+\sum_{A^{\prime} \in \mathcal{K}^{\prime}} \beta\left(A^{\prime}\right)<|f|$ by Claim 4.4 and (4.6), we have $\left|g^{\prime}\right| \leq|f|$ - a contradiction.

Corollary 4.6 Let $\theta^{\prime}=\theta-\frac{1}{2}$ and $\hat{\eta}-\hat{\eta}^{\prime} \leq 1$. Then for all $A \in \mathcal{K}, \beta\left(A^{\prime}\right)=\beta(A)$ and $\hat{\eta}-\hat{\eta}^{\prime}=\frac{1}{2}$.

Proof. Follows from Claim 4.4 and Corollary 4.5 .

Claim $4.7 \theta^{\prime} \neq \theta$.

Proof. Let us assume the contrary. Then for all $A \in \mathcal{K}, \beta\left(A^{\prime}\right) \geq \beta(A)$, for otherwise by Claim 4.2, in $(G, T, \mathcal{K}) g$ can be modified into a multiflow $g^{\prime}$ with $\Theta\left(g^{\prime}\right)>\theta$ - a contradiction. If $\sum_{A^{\prime} \in \mathcal{K}^{\prime}} \beta\left(A^{\prime}\right)>\sum_{A \in \mathcal{K}} \beta(A)$, we have

$$
\theta-\theta^{\prime}=0=\hat{\eta}-\hat{\eta}^{\prime}+\left(\sum_{A^{\prime} \in \mathcal{K}^{\prime}} \beta\left(A^{\prime}\right)-\sum_{A \in \mathcal{K}} \beta(A)\right)>1
$$

a contradiction because $\hat{\eta}>\hat{\eta}^{\prime}$ (otherwise, $g$ is the solution we seek). Then $g[W]=f[W]=$ $\sum_{A \in \mathcal{K}} \beta(A)$ and $\Theta(g)=\Theta(f)$, resulting in $|g|=|f|$ - a contradiction.

Let us call two paths traversing the same inner node $x$ opposite in $x$ if they do not traverse the same edge incident to $x$.

Claim 4.8 Let $x \in N \backslash T$. Then there exists a split of $x$ that decreases $\theta$ by no more than $\frac{1}{2}$.

Proof. Let us assume the contrary. Let the number of paths of $f$ destroyed by a split of $x$ be $n$. Then the split decreases $\Theta(f)$ by at least 1 by Corollary 7.4, thus $8 \geq n \geq 4$. Clearly, 


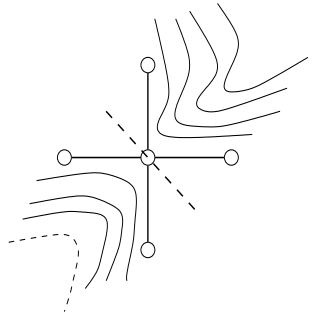

(a)

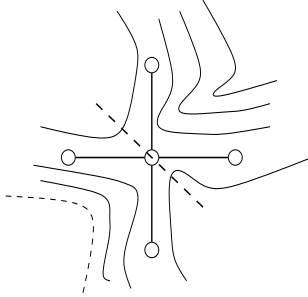

(b)

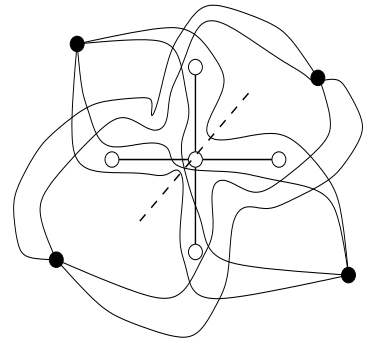

(c)

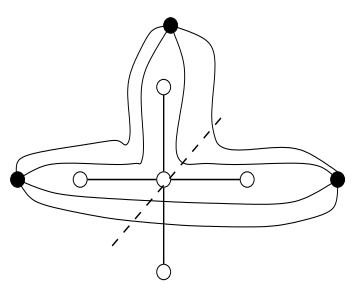

(d)

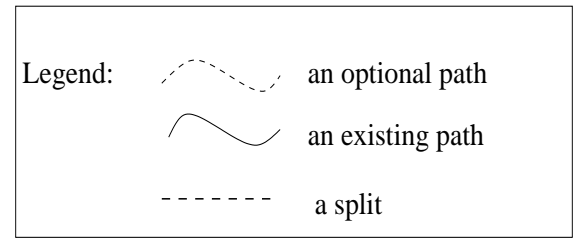

Figure 3: Possible switches of $f$ in an inner node.

$n \neq 7,8$ for otherwise $x$ admits an $f$-split (see Figure 3(a)). Likewise, if $n \in\{5,6\}$, then the switch opposite to the chosen one destroys no more than two paths of $f$ (see Figure $3(b)$ ) - a contradiction. Therefore, $n=4$, and the paths destroyed by a split contribute no more than 1 to $\Theta(f)$. By our assumption, the split decreases $\Theta(f)$ by 1 , and these paths are $S$-paths of $f$ with two common ends. By our assumption, two of these paths cannot be switched so as to comply with the remaining paths traversing $x$. If these two paths are opposite, we switch one pair so as to comply with the other, and there are two options to do so (see Figure 3(c)). The opposite switch affects the other 4 paths of $f$ traversing $x$ and, like above, those paths can traverse $x$ in two different ways. We then select a common switch and obtain a new multiflow $f^{\prime}$ that is a common solution in $(G, T, \mathcal{K})$ and admits an $f^{\prime}$-split in $x$ - a contradiction. If the paths in question are not opposite (see Figure $3(\mathrm{~d})$ ), all the paths of $f$ traversing $x$ end in two terminals. Then there exists a switch of paths of $f$ in $x$ allowing an $f$-split - a contradiction.

We can now finish the proof of the fractionality theorem for the $W$-problem.

Theorem 4.1 Let $(G, T, \mathcal{K})$ be a K-network. Then in $(G, T, \mathcal{K})$ there exists a simple half-integer $W$-problem solution of the smallest size.

Proof. Let $\left(G^{\prime}, T^{\prime}, \mathcal{K}^{\prime}\right)$ be the network with $\theta^{\prime}=\theta-\frac{1}{2}$ and $\hat{\eta}-\hat{\eta}^{\prime} \leq 1$, obtained from $(G, T, \mathcal{K})$ by the maximum number of split-offs in inner nodes. At least one such network exists because of Claim 4.8, By Claim 4.7 and Corollary 4.6, $\beta\left(A^{\prime}\right)=\beta(A)$ for all $A \in \mathcal{K}$. Then $\hat{\eta}-\hat{\eta}^{\prime}=\frac{1}{2}$. Let $g$ denote a simple $W$-problem solution of the smallest size in $(G, T, \mathcal{K})$. Since $|g|=|f|-\frac{1}{2}$, 


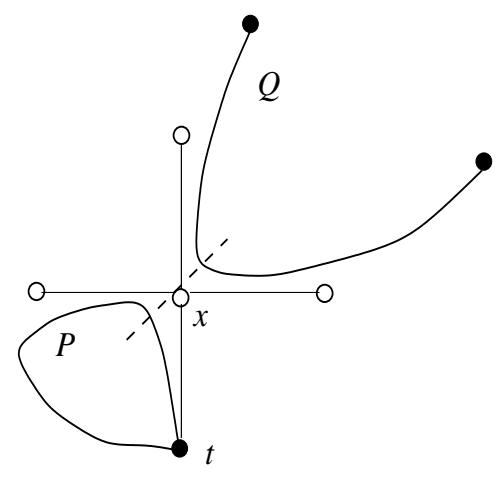

Legend
\begin{tabular}{|ll|}
\hline & an inner node \\
\hdashline & a terminal \\
--- & a path \\
\hline
\end{tabular}

Figure 4: $\theta$-preserving split of an inner node.

$g$ is not maximum and we can add a half-integer zero path $P$ to $g$ with an end in $t \in A$. We select $g$ so that $P$ is the longest w.r.t. number of edges. Let $P$ traverse edge $(t, x)$. Then a path $Q \in g$ opposite to $P$ in $x$ has no end in $t$ (otherwise, switching $P$ and $Q$ prolongs $P$ ).

Switching of $P$ and $Q$ in $x$ cannot increase $g[S]$ for then the resulting half-integer flow $g^{\prime}$ has $\Theta\left(g^{\prime}\right)=\theta$ and $\left|g^{\prime}\right| \leq|f|$. Likewise, switching $P$ and $Q$ so as to allow a $g$-split in $x$ cannot increase $\Theta(g)$, for otherwise we obtain a network $\left(G^{\prime \prime}, T^{\prime \prime}, \mathcal{K}^{\prime \prime}\right)$ with $\theta^{\prime \prime} \geq \theta-\frac{1}{4}$ - a contradiction to Claim 4.7. Therefore, $Q$ is a $t^{c}$-path and an $S$-path. Switching $P$ and $Q$ in $x$ so as to allow a $g$-split of $x$ produces two $W$-paths (see Figure 4). We switch $P$ and $Q$ in this way, obtain a new multiflow $g^{\prime \prime}$ and a network denoted $\left(G^{\prime \prime}, T^{\prime \prime}, \mathcal{K}^{\prime \prime}\right)$. Then $\theta^{\prime \prime}=\theta-\frac{1}{2}$ and $\hat{\eta}^{\prime \prime} \geq \hat{\eta}^{\prime}-\frac{1}{2}=\hat{\eta}-1$ while $\left(G^{\prime \prime}, T^{\prime \prime}, \mathcal{K}^{\prime \prime}\right)$ contains less inner nodes than $\left(G^{\prime}, T^{\prime}, \mathcal{K}^{\prime}\right)$, contrary to our choice.

\section{$5 \quad$ Fractionality of the $S$-problem}

We use Theorem 4.1 to show that the fractionality conjecture for the $S$-problem holds. Let us select a K-network $(G, T, \mathcal{K})$ which is a counterexample to the conjecture,

$$
\text { minimal in fractionality } k \text { and } \alpha:=\frac{\sum_{t \in T}|N(t)|}{|T|} \text {. }
$$

Like in Section 4, we can assume that $k=4$.

Claim $5.1 \alpha=1$

Proof. Let us assume the contrary and select $t \in T$ with $|N(t)| \geq 2$. Let $g$ be a quarter-integer common solution to the $W$ - and $S$-problems in $(G, T, \mathcal{K})$. Let us suppose first that no path of $g$ has an end in $t$. We turn $t$ into an inner node, adding a new terminal $t^{\prime} \sim t$ and an edge 
$\left(t, t^{\prime}\right)$ if $d(t)$ is odd. In the resulting network $\left(G^{\prime}, T^{\prime}, \mathcal{K}^{\prime}\right), \eta^{\prime}:=\eta\left(G^{\prime}, T^{\prime}, \mathcal{K}^{\prime}\right)=\eta$ because the reverse operation does not decrease $\eta^{\prime}$. Let us suppose now that $g$ contains paths with an end in $t$. Let $w_{g}(t)$ denote the total weight of $g$ 's paths beginning in $t$. Then $w_{g}(t) \leq \frac{3}{4} d(t)$, for otherwise there exists an edge $(t, x)$ traversed by four paths of weight $\frac{1}{4}$ with an end in $t$. We replace $(t, x)$ with a new edge $\left(t^{\prime}, x\right)$, where $t^{\prime} \sim t$ is a new terminal, and turn $t$ into an inner node. We also add enough $\left(t, t^{\prime}\right)$-edges to allow the paths of $g$ with an end in $t$ to end in $t^{\prime}$ instead and the degree of $t$ to be even. In the resulting network $\left(G^{\prime}, T^{\prime}, \mathcal{K}^{\prime}\right), \alpha^{\prime}<\alpha$ and $\eta^{\prime}=\eta$ because the reverse operation does not decrease $\eta^{\prime}$.

Theorem 5.2 Every $K$-network $(G, T, \mathcal{K})$ admits a half-integer least-size $W$-problem solution $f$ that also solves the $S$-problem.

Proof. Let $(G, T, \mathcal{K})$ be a K-network $(G, T, \mathcal{K})$. By Claim 5.1, we can transform $(G, T, \mathcal{K})$ into a K-network $\left(G^{\prime}, T^{\prime}, \mathcal{K}^{\prime}\right)$ with $\alpha=1, \eta^{\prime}=\eta$ and $\theta^{\prime}=\theta$. Moreover, every $S$-problem or $W$-problem solution in $\left(G^{\prime}, T^{\prime}, \mathcal{K}^{\prime}\right)$ remains such in $(G, T, \mathcal{K})$ after the reverse transformation. By Theorem 4.1, $\left(G^{\prime}, T^{\prime}, \mathcal{K}^{\prime}\right)$ admits a simple half-integer $W$-problem solution of the smallest size, denoted $f^{\prime}$. By Theorem 1 of [Va 2007], $f^{\prime}$ solves the $S$-problem in $\left(G^{\prime}, T^{\prime}, \mathcal{K}^{\prime}\right)$. Then the multiflow $f$ in $(G, T, \mathcal{K})$, obtained from $f^{\prime}$, solves both $W$ - and $S$-problems.

Corollary 5.3 In a general, not necessarily Eulerian, network $(G, T)$ where the anticlique clutter of $(T, S)$ is a $K$-clutter, both $W$-problem and $S$-problem have fractionality 4.

\section{Acknowledgments}

The author expresses her deepest gratitude to Prof. Eyal S. Shimony for the help with this manuscript and the Lynn and William Fraenkel Center for Computer Science for partially supporting this work.

\section{Appendix: combinatorial max-min for the $W$-problem}

Let $\mathcal{E}=\{\alpha, \beta, \ldots\}$ be a partition of $T$ such that for each $\alpha \in \mathcal{E}$ any $t^{\prime}, t^{\prime \prime} \in \alpha$ are equivalent (an equi-partition). We call $\mathcal{X}=\left(X_{\alpha}: \alpha \in \mathcal{E}\right)$ is an expansion if $X_{\alpha} \cap T=\alpha, \alpha \in \mathcal{E}$. Taking members of $\mathcal{X}$ as terminals and an induced clutter, we obtain a new network with a 
graph $G_{\mathcal{X}}$, terminals $\mathcal{X}$ and a clutter $\mathcal{K}_{\mathcal{X}}$ on $\mathcal{X}\left(\mathcal{K}_{\mathcal{X}}\right.$ is a K-clutter if $\mathcal{K}$ is a K-clutter $)$. For $X_{\alpha}, X_{\beta} \in \mathcal{X}$, we call $\left(X_{\alpha}, X_{\beta}\right)$ strong or weak if for every $s \in \alpha$ and $t \in \beta,(s, t) \in S$ or $(s, t) \in W$ respectively. Likewise, $X_{\alpha} \sim X_{\beta}$ if for every pair of terminals $s \in \alpha$ and $t \in \beta$, $s \sim t$. An $\mathcal{X}$-path in $G$ is an $(x, y)$-path with $x, y$ lying in distinct members of $\mathcal{X}$. An $\mathcal{X}$-flow is a flow in the network $\left(G_{\mathcal{X}}, \mathcal{X}, \mathcal{K}_{\mathcal{X}}\right)$ consisting of $\mathcal{X}$-paths. The $S$-problem and the $W$-problem in $\left(G_{\mathcal{X}}, \mathcal{X}, \mathcal{K}_{\mathcal{X}}\right)$ are defined in the same way as for $(G, T, \mathcal{K})$, and their maxima are denoted by $\eta_{\mathcal{X}}$ and $\theta_{\mathcal{X}}$ respectively.

We define a partial order on expansions as follows. Let $\mathcal{E}$ and $\mathcal{F}$ be equi-partitions of $T$ and let $\mathcal{X}=\left(X_{\alpha}: \alpha \in \mathcal{E}\right)$ and $\mathcal{Y}=\left(Y_{\alpha}: \alpha \in \mathcal{F}\right)$ be expansions. Then $\mathcal{X} \preceq \mathcal{Y}$ if for every $X \in \mathcal{X}$ there exists $Y \in \mathcal{Y}$ so that $X \subset Y$. Note that for every $\mathcal{X} \preceq \mathcal{Y}$, every $\mathcal{X}$-flow is also a $\mathcal{Y}$-flow (but the converse may be not true). Since for $\mathcal{X} \preceq \mathcal{Y}$ any $\mathcal{X}$-flow is also a $\mathcal{Y}$-flow, $\theta_{\mathcal{Y}} \geq \theta_{\mathcal{X}}$. Since $T$-flow is also an $\mathcal{X}$-flow, $\theta_{\mathcal{X}} \geq \theta$. $\mathcal{X}$ is called critical if $\theta_{\mathcal{Y}}>\theta_{\mathcal{X}}$ for every $\mathcal{Y} \succ \mathcal{X}$. A critical $\mathcal{X}$ with $\theta_{\mathcal{X}}=\theta$ is called a dual solution. The triangle theorem ([L 1985]) ensures that:

$$
\text { there exists a maximum } \mathcal{X} \text {-flow } h \text { such that } \Theta_{\mathcal{X}}(h)=\theta_{\mathcal{X}} \text {. }
$$

We limit ourselves to networks $(G, T, \mathcal{K})$ with simple $\mathcal{K}$. The results of this section that hold for simple clutters hold for general networks as well, because compressing a non-trivial atom into one terminal does not change $\theta$ by triangle theorem from [L 1985] and metric properties of a K-clutter. For a K-network with simple $\mathcal{K}$, every subset in an expansion $\mathcal{X}$ contains exactly one terminal; $X_{t}$ denotes a member of $\mathcal{X}$ containing $t \in T$. Then (7.7) implies that for a maximum $X$-flow $h$ (even when $\mathcal{X}=T)$ :

$$
\Theta_{\mathcal{X}}(h)=|h|-\frac{1}{2} h[W] .
$$

We aim to prove the following max-min theorem for the fractional $W$-problem.

Theorem 7.1 In a K-network $(G, T, \mathcal{K})$ :

$$
\max _{f} \Theta(f)=\min _{\mathcal{X}}\left(\frac{1}{2} \sum_{t \in T} d\left(X_{t}\right)-\frac{1}{2} \sum_{A \in \mathcal{K}_{\mathcal{X}}} \beta(A)\right) .
$$

The maximum is taken over the fractional multiflows in $(G, T, \mathcal{K})$, and the minimum is taken over all expansions in $(G, T, \mathcal{K})$. Moreover, (7.9) holds as equality for every dual solution $\mathcal{X}$.

To prove this theorem, we state the following inequality for an expansion $\mathcal{X}$ and a $T$-flow $f$ :

$$
\Theta(f) \leq \theta \leq \Theta_{\mathcal{X}}(h) \leq \frac{1}{2} \sum_{t \in T} d\left(X_{t}\right)-\frac{1}{2} \sum_{A \in \mathcal{K}_{\mathcal{X}}} \beta(A)
$$


We aim to show that (7.10) holds as inequality for every expansion and as equality for every critical expansion. (7.10) (a) follows directly from the definition of $\theta$. (7.10) (b) holds because $f$ is also an $\mathcal{X}$-flow. (7.10) (c) holds because there exists a maximum $\mathcal{X}$-flow $h$ that solves the $W$-problem in $\mathcal{X}$. For such $h$ the minimum of $\sum_{A \in \mathcal{K}_{\mathcal{X}}} h[A]$ is achieved when all $A \in \mathcal{K}_{\mathcal{X}}$ are locked by $h$, i.e. $\sum_{A \in \mathcal{K}_{\mathcal{X}}} h[A] \leq \sum_{A \in \mathcal{K}_{\mathcal{X}}} \beta(A)$ and $|h|=\frac{1}{2} \sum_{t \in T} \lambda\left(X_{t}\right)$ by the Lovãsz-Cherkassky theorem ([Lo 1976, Ch 1977]). We need the following two claims to show that (17.10) (c) is an equality.

Claim 7.2 Let $(G, T, \mathcal{K})$ be a simple K-network, and let $\mathcal{X}$ be a dual solution in it. A maximum fractional $\mathcal{X}$-flow $h$ that satisfies $\Theta_{\mathcal{X}}(h)=\theta_{\mathcal{X}}$ (that is, solves the $W$-problem in $\left.\left(G_{\mathcal{X}}, \mathcal{X}, \mathcal{K}_{\mathcal{X}}\right)\right)$ locks $X_{t}$ for all $t \in T$.

Proof. First, let us show that $h$ saturates every $\left(X_{t}, \overline{X_{t}}\right)$-edge. Let $e$ be an $(x, y)$-edge with $x \in X_{t}$ and $y \in \overline{X_{t}}$. Let $\mathcal{Y} \succ \mathcal{X}$ be an expansion where $Y_{s}=X_{s}$ for terminal $s \neq t$ and $Y_{t}=X_{t} \cup\{y\}$. Since $\mathcal{X}$ is critical, $\theta_{\mathcal{Y}}>\theta_{\mathcal{X}}$ and there exists a $\mathcal{Y}$-flow $g$ such that $\Theta_{\mathcal{Y}}(g)>\theta_{\mathcal{X}}$. Let us denote the unused capacity of $e$ by $\varepsilon$ and let $\delta=g\left[y, \cup_{s \neq t} X_{s}\right]$. Clearly, $\varepsilon<\delta$. We turn $g$ into an $\mathcal{X}$-flow by prolonging all its paths starting in $y$ to $x$ instead through the edge $e$. Let $g^{\prime}$ be the functions on $\mathcal{X}$-paths thus obtained; $g^{\prime}$ does not satisfy the capacity constraint on $(x, y)$. Then there exists $0<\alpha<1$ such that $h^{\prime}=(1-\alpha) h+\alpha g^{\prime}$ is an $\mathcal{X}$-flow. $h^{\prime}$ satisfies all capacity constraints and has $\Theta_{\mathcal{X}}\left(h^{\prime}\right) \geq(1-\alpha) \Theta_{\mathcal{X}}(h)+\alpha \Theta_{\mathcal{Y}}(g)>\theta_{\mathcal{X}}$, contradicting the definition of $\mathcal{X}$. Let us assume now that a $(p, q)$-path $P$ of $h, p \in X_{t}$, contains two $\left(X_{t}, \overline{X_{t}}\right)$-edges, $e_{1}=\left(x_{1}, y_{1}\right)$ and $e=\left(x_{2}, y_{2}\right)$ where $x_{1}, x_{2} \in X_{t}, y_{1}, y_{2} \in \overline{X_{t}}$ and $y_{1}, x_{1}, x_{2}, y_{2}$ appear on $P$ in this order. Then by replacing $P$ with $x_{2} P q$ we obtain an $\mathcal{X}$-flow $g$ for which $\Theta_{\mathcal{X}}(g)=\theta_{\mathcal{X}}$ and the edge $\left(x_{1}, y_{1}\right)$ is not saturated by $g$, a contradiction.

Claim 7.3 Let $(G, T, \mathcal{K})$ be a simple K-network, and let $\mathcal{X}$ be a dual solution. A maximum fractional $\mathcal{X}$-flow $h$ would then satisfy $\Theta_{\mathcal{X}}(h)=\theta_{\mathcal{X}}$ iff every $A \in \mathcal{K}_{\mathcal{X}}$ is locked by $h$.

Proof. The "if" direction is trivial. Let $h$ be a maximum $\mathcal{X}$-flow with $\Theta_{\mathcal{X}}(h)=\theta_{\mathcal{X}}$ that locks every member of $\mathcal{K}_{\mathcal{X}}$. Because of Claim 7.2 and the simplicity of $\mathcal{K}_{\mathcal{X}}$, we get $\Theta(h)=$ $\frac{1}{2} \sum_{X \in \mathcal{X}} d(X)-\frac{1}{2} \sum_{A \in \mathcal{K}_{\mathcal{X}}} \beta_{A}$ and thus $\Theta(h) \geq \theta_{\mathcal{X}}$ by $(\overline{7.10})(\mathrm{c})$.

For the "only if" direction, assume that $h$ is a maximum $\mathcal{X}$-flow that has $\Theta_{\mathcal{X}}(h)=\theta_{\mathcal{X}}$ and unlocks $A \in \mathcal{K}_{\mathcal{X}}$. Let $A^{c}$ in the context of $\mathcal{K}_{\mathcal{X}}$ denote the members of $\mathcal{X}$ that do not lie in $A$. Then $h$ contains an augmenting sequence $P_{0}, x_{0}, \ldots, x_{m-1}, P_{m}$, where $P_{0}$ is an $A$-path, $P_{m}$ is 


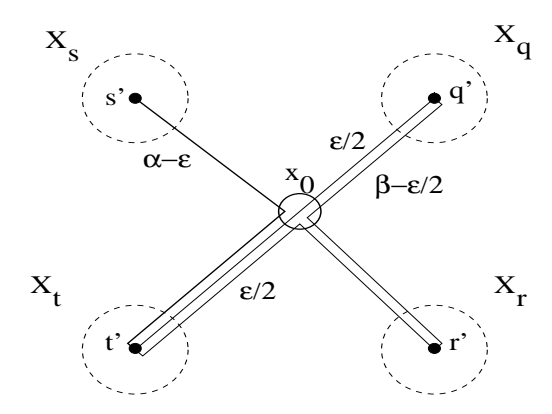

Figure 5: The fractional $\frac{3}{2}$-operation.

an $A^{c}$-path, and each one of $P_{1}, \ldots, P_{m-1}$ is an $\left(A, A^{c}\right)$-path. We can choose $h$ so that $m=1$. Let $P_{0}$ and $P_{1}$ be $\left(s^{\prime}, t^{\prime}\right)$ - and $\left(q^{\prime}, r^{\prime}\right)$-paths with weights $\alpha$ and $\beta$ respectively where $s^{\prime} \in X_{s}$, $t^{\prime} \in X_{t}, q^{\prime} \in X_{q}$ and $r^{\prime} \in X_{r}$. Since a switch of $P_{0}$ and $P_{1}$ in $x_{0}$ cannot increase $\Theta(h)$, we can assume that w.l.o.g. $\left(X_{q}, X_{r}\right),\left(X_{t}, X_{r}\right)$ and $\left(X_{t}, X_{q}\right)$ are $S$-pairs while $\left(X_{s}, X_{q}\right)$ and $\left(X_{s}, X_{r}\right)$ are $W$-pairs by the simplicity of $\mathcal{K}_{\mathcal{X}}$.

We construct a new flow $f$ from $h$ by replacing $P_{0}$ and $P_{1}$ with $\left(t^{\prime}, r^{\prime}\right),\left(t^{\prime}, q^{\prime}\right),\left(q^{\prime}, r^{\prime}\right)$ and $\left(s^{\prime}, t^{\prime}\right)$-paths of weights $\frac{\varepsilon}{2}, \frac{\varepsilon}{2}, \beta-\frac{\varepsilon}{2}$ and $\alpha-\varepsilon$ respectively (this is the $\frac{3}{2}$-operation, see Figure 5). It follows that $|f|=|h|-\frac{\varepsilon}{2}$ and $f[W]=h[W]-\varepsilon$ since $\left(X_{q}, X_{t}\right),\left(X_{q}, X_{r}\right),\left(X_{r}, X_{t}\right) \in S$ and $\Theta_{\mathcal{X}}(f)=\Theta_{\mathcal{X}}(h)$.

The subpath $s^{\prime} P_{0} x_{0}$ does not have common nodes with any other $\mathcal{X}$-path $Q$ whose ends do not lie in $X_{s} \cup X_{t}$. If it were so, then the above $\frac{3}{2}$-operation could be applied to both $P_{0}, P_{1}$ and $P_{0}, Q$ and a flow $f^{\prime}$ with $\left|f^{\prime}\right|=|h|-\frac{\varepsilon}{2}$ and $f^{\prime}[W]=h[W]-2 \varepsilon$ could be created, which contradicts the maximality of $\Theta_{\mathcal{X}}(h)$. Therefore, there exists an edge $\left(s^{\prime}, x\right)$ of $s^{\prime} L v$ which is not saturated by $f$ - a contradiction to Claim 7.2 .

Theorem 7.1 follows from Claims 7.2 and 7.3 .

Corollary $7.42 \theta(G, T, \mathcal{K}) \in \mathbb{N}$.

Proof. Let $\mathcal{X}$ be an expansion that achieves equality in Theorem 7.1 for $(G, T, \mathcal{K})$. Then $\theta(G, T, \mathcal{K})=\frac{1}{2} \sum_{X \in \mathcal{X}} d(X)-\frac{1}{2} \sum_{A \in \mathcal{K}_{\mathcal{X}}} \beta(A)$, while $\sum_{X \in \mathcal{X}} d(X)$ is always even in an Eulerian network and every $\beta(A)$ is an integer by definition. Thus, a split of an inner node in $(G, T, \mathcal{K})$ decreases $\theta$ by $\frac{k}{2}, k \in \mathbb{N} \cup\{0\}$.

Corollary 7.5 Let $(G, T, \mathcal{K})$ be a simple $K$-network and let $h$ be a simple $W$-problem solution in $(G, T, \mathcal{K})$ with $\sum_{A \in \mathcal{K}} h[A]=\sum_{A \in \mathcal{K}} \beta(A)$. Then $2 h[S] \in \mathbb{N}$.

Proof. $2 h[S]$ is an integer because $\theta=h[S]+\frac{1}{2} h[W]=h[S]+\frac{1}{2} \sum_{A \in \mathcal{K}} \beta(A)$ and $\theta$ is half-integer 


\section{References}

[Ch 1977] B. V. Cherkassky, "Solution of a problem on multicommodity flows in a network," Ekon. Mat. Metody (Russian), vol. 13, pp. 143-151, 1977.

[F 1990] A. Frank, "Packing Paths, Circuits and Cuts - a Survey," in: B. Korte, L. Lovãsz, H. J. Prömel, A. Schrijver (Eds.): Paths, Flows, and VLSI-Layout, Springer, Berlin, 1990.

[IKL 2000] H. Ilani, E. Korach, M. Lomonosov, "On extremal multiflows," J. Comb. Theory Ser. B vol. 79, pp. 183-210, 2000.

[K 1989] A. Karzanov, "Polyhedra related to undirected multicommodity flows", Linear Algebra and its Applications, vol. 114-115 pp. 293-328, 1989.

[KL 1978] A. Karzanov and M. Lomonosov, "Systems of flows in undirected networks," Math. Programming. Problems of Social and Economical Systems. Operations Research Models. Work collection. Issue 1 (Russian), Moscow, pp. 59-66, 1978.

[L 1985] M. Lomonosov, "Combinatorial approaches to multiflow problems", Appl. Discrete Math. 11, No. 1, pp. 1-93, 1985.

[L 2004] M. Lomonosov, "On return path packing," European Journal of Combinatorics, Vol. 25, No. 1, pp. 35-53, 2004.

[Lo 1976] L. Lovãsz, "On some connectivity properties of Eulerian graphs," Acta Math. Akad. Sci. Hungaricae, vol. 28, pp. 129-138, 1976.

[Va 2007] N. Vanetik, "Path packing and a related optimization problem", to appear in Journal of Combinatorial Optimization, 2007. 
Keywords

Path packing, multiflow, fractionality 


\section{Contact author}

Natalia Vanetik

Department of Computer Science, Ben-Gurion University, Israel.

E-mail address: orlovn@cs.bgu.ac.il

Fax: $+972-8-6477650$

Phone: +972-8-6477866

Address:

N. Vanetik

Department of Computer Science

Ben Gurion University of the Negev

P.O.B 653 Be'er Sheva 84105

Israel 


\section{Footnotes}

\section{Author affiliation:}

N. Vanetik, Department of Computer Science, Ben-Gurion University, Israel, orlovn@cs.bgu.ac.il 